\title{
Immediate memory for visual patterns: Symmetry and amount of information '
}

\author{
M. M. SCHNORE, UNIVERSITY OF WESTERN ONTARIO AND ONTARIO HOSPITAL, ST. THOMAS \\ J. T. PARTINGTON, ALCOHOLISM AND DRUG ADDICTION RESEARCH FOUNDATION, LONDON, ONTARIO
}

Immediate recall for visual patterns which varied in degree of symmetry and amount of information (2.6 to 13.7 bits) was studied. It was found that recall errors were primarily a linear function of degree of symmetry and amount of information.

A well known proposition of Gestalt psychology states that "good" or symmetrical figures are remembered more accurately than "poor" or asymmetrical ones because the trace resulting from a good figure is more stable. Several investigators (e.g., Michels \& Zusne, 1965) have argued, however, that in terms of information theory, symmetry may be considered to be a form of redundancy. In information theory terms, it is easier to remember symmetrical as opposed to asymmetrical patterns because the former contain less information. Attneave (1955), using dot patterns as stimuli, found that symmetrical patterns were reproduced from memory more accurately only when they contained less information than asymmetrical patterns. It should be noted that in the present context "redundancy" refers to the amount of constraint employed in the construction of patterns. This differs from Garner's (1962) definition of redundancy which refers to figural complexity.

In the present study the generality of Attneave's findings were investigated by studying immediate recall of patterns which contained less information than those used by Attneave. The amount of information of the patterns used in this study varied from 2.6 to 13.7 bits, while Attneave employed patterns with 12 to 35 bits of information. In general, the method of this study is similar to that employed by Attneave; however, several methodological improvements, which are noted below, were incorporated.

Method

The stimuli were 4 by 4 square matrices each consisting of eight black and eight white cells. The decision to use black squares instead of dots was prompted by the observation that dot patterns are at times perceived as separate clusters of unrelated dots, especially when the proximity of the dots increases. A sample of four types of patterns with varying degrees of symmetry is presented in Fig. 1. For Pattern A, the occurrence of a black or white cell was determined randomly for the four cells in the upper left quadrant, with the constraint that two of the cells had to be black. The remaining quadrants of the pattern were obtained by reflecting vertically and then horizontally the quadrant for which the nature of the cells was determined randomly. Thus, Pattern A was symmetrical vertically as well as horizontally with the axes of symmetry passing between the second and third column and row. For Pattern $B$ the nature of the eight cells in the upper two quadrants were determined randomly, while for Pattern C, 12 cells were random. The remaining quadrants in each pattern, as for Pattern A, were obtained by reflection. In Pattern $D$ the occurrence of a black or white cell was determined randomly for all 16 cells, therefore, these patterns were the least symmetrical. Only patterns containing eight black and eight white cells were used in this study, in view of Attneave's (1955) finding that the number of errors in recall were positively correlated with the number of dots in a pattern.

In terms of information theory, type A patterns may be said to contain 2.6 bits of information because only six distinct patterns can be constructed under the rules outlined above $\left(\frac{4 !}{2 ! 2 !}=6 ; \log _{2} 6=2.6\right.$ bits $)$. The three quadrants of Pattern A which were obtained by reflection may be considered to be redundant and not adding any further uncertainty. By analogous reasoning, $B, C$, and $D$ patterns can be said to contain approximately $6.1,9.9$, and 13.7 bits of information, respectively. In terms of amount of redundancy, $A, B$, $\mathrm{C}$, and $\mathrm{D}$ patterns may be considered to be $84 \%, 62 \%$. $38 \%$, and $15 \%$ redundant in comparison to the maximum uncertainty that is associated with a 4 by 4 matrix pattern without any constraints (Redundancy $=1-\frac{\mathrm{H}_{\mathrm{a}}}{\mathrm{H}_{\max }}$ x 100; where $\mathrm{H}_{\mathrm{a}}$ =actual amount of uncertainty in a

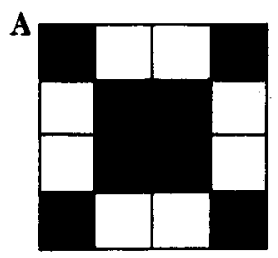

$2.6 \mathrm{sITS}$

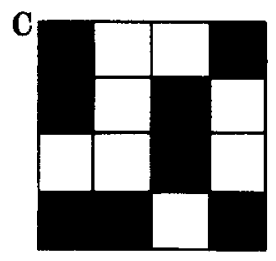

$9.9 \mathrm{BITs}$

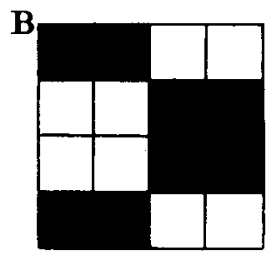

$6.1 \mathrm{BITS}$

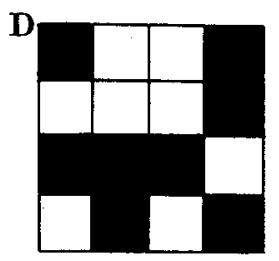

13.7 BITS
Fig. 1. A sample of the stimuli containing different amounts of information. 


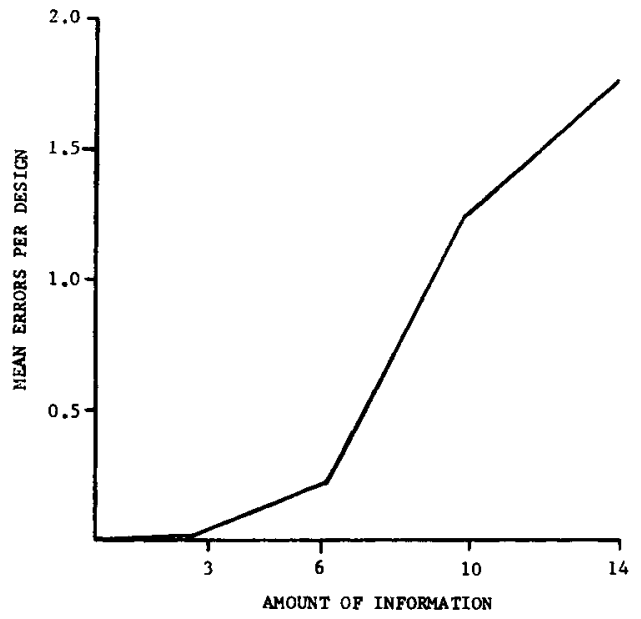

Fig. 2. Mean errors per design as a function of the amount of information.

pattern and $\mathrm{H}_{\max }=$ maximum amount of uncertainty, i.e., 16 bits).

The Ss were 214 university summer school students, mostly teachers, with a mean age of 31 years. Altogether there were 30 patterns, six of Type A and eight each of Types B, C, and D. Each pattern was exposed on a screen (size of the image was approximately 35 in. $x 35$ in.) by an automatic projector for $2.5 \mathrm{sec}$ and $\mathrm{Ss}$ had $10 \mathrm{sec}$ to reproduce a pattern immediately after it was removed. Testing was carried out in classrooms for groups of approximately $50 \mathrm{Ss}$ each, which allowed partial counterbalancing of the order of presentation of patterns.

The Ss attempted to reproduce the patterns by indicating the location of black cells on response sheets, each of which contained an empty 4 by 4 matrix. Responses were scored in terms of the number of cells reproduced incorrectly (white and black), yielding a range of possible errors from 0 to 16 per pattern. Results

Mean errors for the four types of patterns which varied in symmetry and amount of information (2.6, $6.1,9.9$, and $13.7 \mathrm{bits})$ were: $.02, .20,1.23$, and 1.76 . These results are presented graphically in Fig. 2. Analysis of variance showed that the differences in the error scores were statistically significant ( $F=$ 460.41 , $\mathrm{df}=3 / 639, \mathrm{p}<.01$ ). Individual comparisons of mean errors revealed that all the differences among the four types of patterns were also significant $(p<.05)$. A trend analysis (Ferguson, 1965) showed that the relationship between information content of the stimuli and recall performance was primarily linear (Monotonic, $z=24.01, p<.01$ ) and to a lesser degree cubic (Tritonic, $z=6.16, p<.01$ ). Finally, the correlation between age and total recall errors was significant $(r=+.36, p<.01)$, while sex was not related to recall performance.

\section{Discussion}

The main purpose of this study was to examine more closely the relationship between information content of stimuli and recall performance. This relationship was found to be primarily linear, which indicates that recall errors are a positive function of the information content of stimuli. The additional cubic component of this relationship suggests a relatively greater change in recall performance somewhere between the 6 and 10 bit levels of stimulus information. The present findings are in agreement with those of Attneave (1955), who also found a positive relationship between the information content of dot patterns and recall errors.

The fact that practically no errors were made on the 2.6 and 6.7 bit designs suggests that the information content of these designs was within the Ss' channel capacity. The increased errors for the 9.9 and 13.7 bit designs, on the other hand, suggests that the information content of these designs somewhat exceeded the Ss' channel capacity.

Another purpose of this experiment was to improve upon the testing method previously used to study the relationship between information content of stimuli and memory performance. The visual patterns developed for the present experiment were relatively less complex than those used in former studies. The method of designing stimuli used in the present experiment appears promising for several reasons. First, the approximate order of recall difficulty for a set of such stimulus patterns may be established without tedious empirical item analyses. This point is supported by two sets of findings: (1) the obtained linear relationship between information content of stimuli and recall performance, and (2) an item analysis of the present set of stimuli which showed that only three of the 30 patterns were unacceptable in terms of discriminating power (i.e., performance on the item vs total score). A second reason for confidence in the potential value of the present test format is suggested by some tentative evidence of discriminant validity. Specifically, age but not sex was related to recall performance, and since the age variable is often conceptually linked with memory, while there is no such expected association between memory and sex, these data suggest some validational support for this method of assessing memory functioning.

\section{References}

Attneave, F. Symmetry, information, and memory for patterns. Amer. J. Psychol., 1955, 68, 209-222.

Ferguson, G. A. Nonparametric trend analysis. Montreal: McGill University Press, 1965.

Gamer, W. R. Uncertainty and structure as psychological concepts. New York: Wiley and Sons, 1962.

Michels, K. M., \& Zusne, L. Metrics of visual form. Psychol. Bull., $1965,63,74-86$.

\section{Note}

1. This study was assisted under Grant No. 57 of The Ontario Mental Health Foundation. 